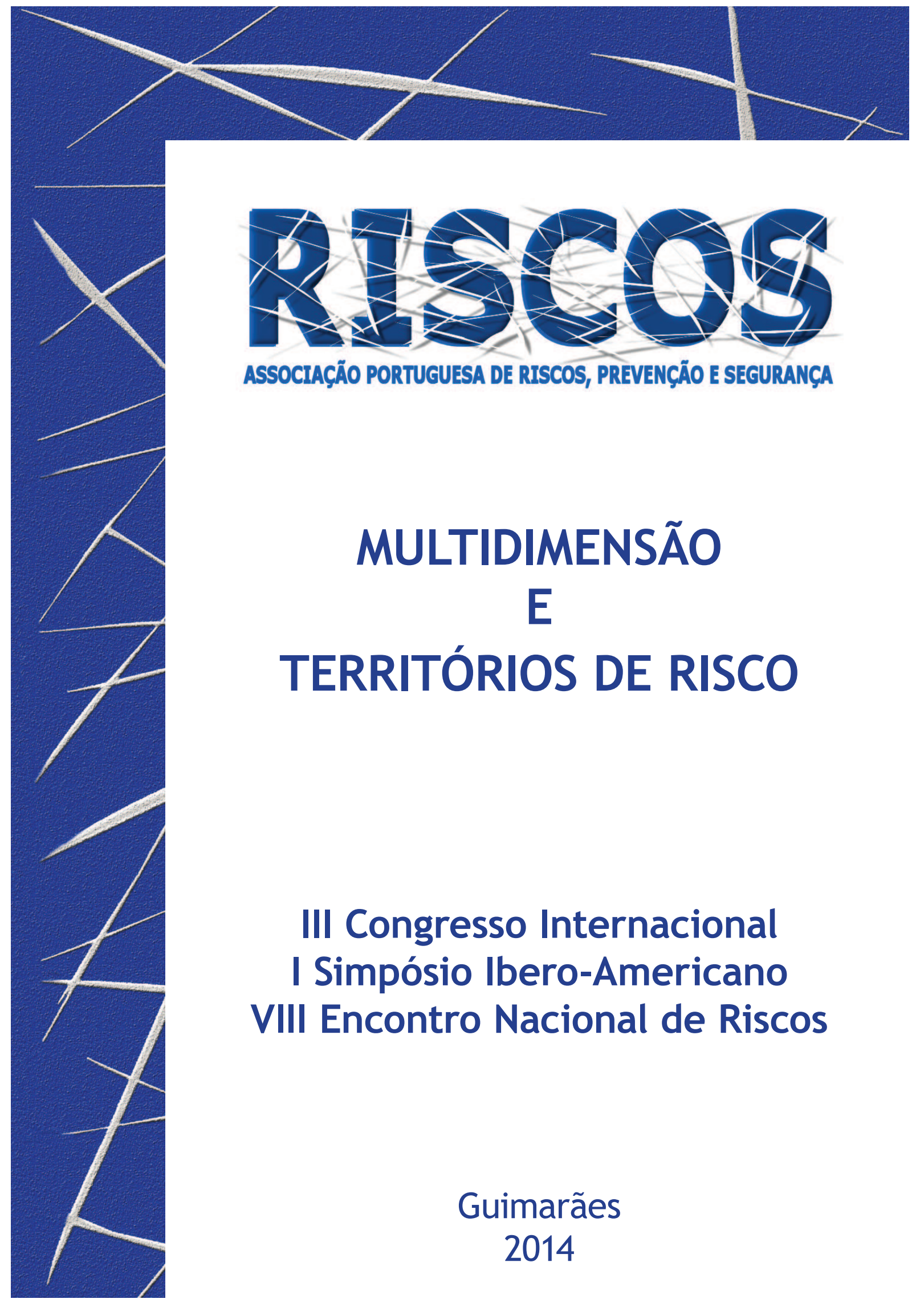




\title{
RISCOS TECNOLÓGICOS: MODELAÇÃO E AVALIAÇÃO DAS CONSEQUÊNCIAS DE UM ACIDENTE NO TRANSPORTE RODOVIÁRIO DE CLORO
}

\author{
Henrique Costa \\ Faculdade de Ciências da Universidade do Porto \\ hvcosta@hotmail.com \\ Manuel Trelles \\ Faculdade de Ciências da Universidade do Porto \\ manuel77trelles@gmail.com \\ António Gomes \\ CEGOT - Faculdade de Letras da Universidade do Porto \\ atgomes@letras.up.pt
}

\section{RESUMO}

O presente trabalho analisa um acidente tecnológico ocorrido durante o transporte rodoviário de uma cisterna com 21 toneladas de cloro $\left(\mathrm{Cl}_{2}\right)$. Apesar de não ter ocorrido fuga da substância transportada, são avaliadas as potenciais consequências para a população e ambiente, caso a fuga de cloro tivesse ocorrido. Explana-se os softwares utilizados para a modelação da possível área atingida pela fuga, e ainda para determinar o número e as possíveis consequências fisiológicas na população presente das áreas afetadas.

Palavras-chave: Riscos Tecnológicos; SIG; Cloro; Matérias Perigosas; Proteção Civil;

Introdução

A 30 de Julho de 2009 ocorreu um fatídico acidente na freguesia de Gondarém, concelho de Vila Nova de Cerveira. Um camião que transportava 21 toneladas de cloro (.$^{\circ}{ }^{\circ}$ ONU 1017), despistouse no viaduto de acesso à A28, precipitando-se para a EN13 que se encontra a uma cota inferior. Deste acidente não ocorreu a fuga do produto transportado, contudo importa aferir quais seriam as áreas e a população potencialmente afetada caso esta tivesse ocorrido. Esta análise assume-se como um contributo para melhorar a prevenção, resposta e mitigação de futuros acidentes deste tipo.

\section{Propriedades do Cloro}

O cloro é um gás corrosivo, tóxico e comburente (ANPC, 2011), mais denso que o ar (tabela I), tendo sido inclusivamente utilizado como arma de guerra química durante primeira guerra mundial (Winder, 2001) dadas as suas propriedades toxicológicas (tabela II).

\section{Tabela I - Propriedades do Cloro}

\begin{tabular}{|cc|} 
Propriedade & Valores \\
\hline Peso atómico & 35.43 \\
Peso molecular & 70.91 \\
Ponto de ebulição & $-34.6^{\circ} \mathrm{C}$ \\
Ponto de fusão a $760 \mathrm{mg} \mathrm{Hg}$ & $-101^{\circ} \mathrm{C}$ \\
Pressão de vapor a $0^{\circ} \mathrm{C}$ e a $760 \mathrm{mg} \mathrm{Hg}$ & 3.61 a $760 \mathrm{mg} \mathrm{Hg}$ \\
Densidade a $0^{\circ} \mathrm{C}$ e a $760 \mathrm{mg} \mathrm{Hg}$ & $3.21 \mathrm{~g} / \mathrm{L}$ \\
\hline Solubilidade em água a $0^{\circ} \mathrm{Ce}$ a $760 \mathrm{mg} \mathrm{Hg}$ & $14.6 \mathrm{~g} / \mathrm{L}$ (diminui com o aumento da temperatura) \\
\hline
\end{tabular}

Fonte: Winder, 2001 
CAPÍTULO 3.4: RISCOS TECNOLÓGICOS E DESENVOLVIMENTO

Tabela II - Efeitos no ser humano da exposição ao cloro

\begin{tabular}{cc} 
Concentração & Efeitos no ser humano \\
\hline $1-3 \mathrm{ppm}$ & Ligeira irritação das membranas mucosas que pode ser tolerado durante curtos períodos de \\
& tempo. Referência para o limite de exposição de curta duração 3 ppm. \\
$>5 \mathrm{ppm}$ & Irritação dos olhos \\
$>15 \mathrm{ppm}$ & Irritação da garganta \\
15 a $30 \mathrm{ppm}$ & Tosse, asfixia, queimaduras \\
$>50 \mathrm{ppm}$ & Pneumonite química \\
$430 \mathrm{ppm}$ & Morte após 30 minutos de exposição \\
$>1000 \mathrm{ppm}$ & Morte em poucos minutos \\
\hline
\end{tabular}

Fonte: Adaptado de Winder (2001) e Evans (2005)

\section{Metodologia}

A modelação das áreas potencialmente afetadas pela fuga de Cloro foi realizada com o software ALOHA - Areal Locations of Hazardous Atmospheres - (EPA \& NOAA, 2007), tendo em conta os Inputs especificados na figura 1. As condições meteorológicas utilizadas na modelação foram as observadas no dia e hora do acidente, registadas pelo SNIRH (Sistema Nacional de Informação de Recursos Hídricos), concretamente na estação meteorológica de Vila Nova de Cerveira.

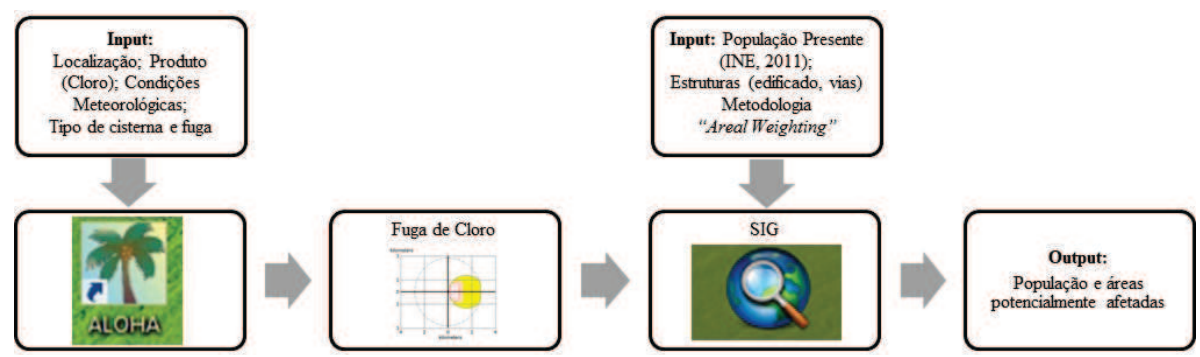

Figura 1- Metodologia da modelação do acidente tecnológico

Para se estimar no ArcMap a população e as áreas potencialmente afetadas face à exposição da fuga de cloro, foi utilizado o método de ponderação espacial "Areal Weighting”, de acordo com o descrito por Chakraborty \& Amostrong (1995) e Margai (2001), em que:

População potencialmente afetada $=\sum_{i=0}^{n} P i+\sum_{j=0}^{m}\left(P j * \frac{a_{j^{\prime}}}{a_{j}}\right)$

$\mathrm{n}=\mathrm{n} \cdot{ }^{\circ}$ de subsecções que se encontram totalmente contidas na área do sinistro (cujos limites não coincidem com os limites da área afetada);

$\mathrm{Pi}=$ população das subsecções totalmente contidas na área do sinistro e em que $i=0,1,2, \ldots, n$; $\mathrm{m}=\mathrm{n} .{ }^{\circ}$ de subsecções parcialmente contidas na área do sinistro (cujos limites intersectam ou coincidem com o limite da área afetada);

$\mathrm{Pj}$ = população das subsecções parcialmente contidas na área do sinistro em que $\mathrm{j}=0,1,2, \ldots$, $\mathrm{m}$;

aj = Área total das subsecções contidas parcialmente na área do sinistro;

$a^{\prime}{ }^{\prime}$ = Área das subsecções parcialmente contidas na área do sinistro;

Pela hora e dia da ocorrência do acidente (cerca das $14 \mathrm{~h} 00$ de sexta-feira), foram realizados os cálculos da população potencialmente afetada tendo em conta a População Presente, por subsecção, à data dos censos 2011 do INE. 

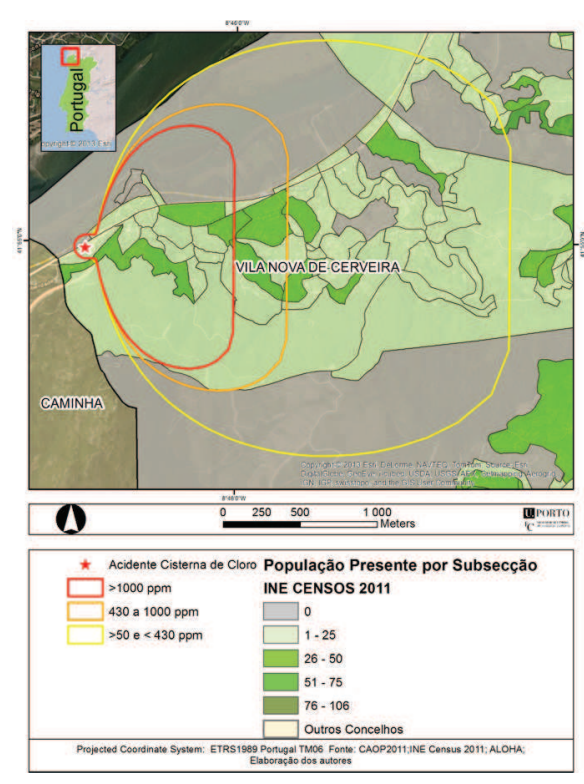

Figura 2 - Dispersão da fuga de cloro e População Presente por subsecção
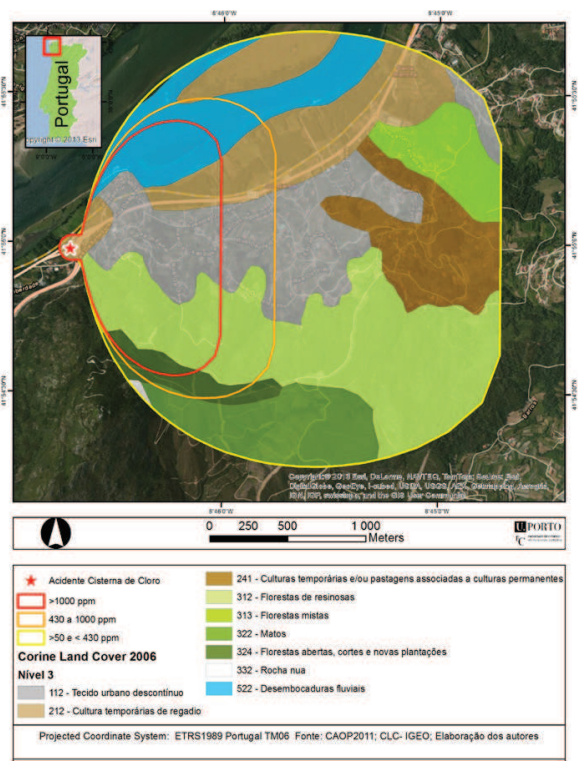

Figura 3 - Dispersão da fuga de cloro e uso do solo (Corine Land Cover 2006)

Tabela IV - População potencialmente afetada

\begin{tabular}{cccc}
\hline Concentração & Consequência & $\begin{array}{c}\text { Área } \mathbf{k m}^{2} \text { Total de População Presente } \\
\text { potencialmente afetada }\end{array}$ \\
\hline$>1000 \mathrm{ppm}$ & Morte em poucos minutos & 1,18 & 237 \\
$430 \mathrm{ppm}$ & Morte em 30 min. de exposição & 0,74 & 133 \\
$>50 \mathrm{ppm}$ & Pneumonite química, tosse, irritação de garganta e olhos, etc. & 4,09 & 525 \\
& Total & 6,01 & 896 \\
\hline
\end{tabular}

Os resultados obtidos na modelação patente na figura 2 e tabela IV evidenciam que a área mortal em poucos minutos (>1000ppm), atingiria uma superfície de $1,18 \mathrm{~km}^{2}$, onde 237 indivíduos seriam potencialmente afetados. Também 133 indivíduos poderiam vir a falecer caso estivessem expostos durante 30 minutos a 430ppm, e 525 iriam sofrer consequências severas para a saúde como pneumonite química.

Tabela V - Área potencialmente afetada por tipo de uso do solo

\begin{tabular}{ccc}
\hline Código CLC & $\begin{array}{c}\text { Tipo de uso do solo } \\
\text { (Corine Land Cover Nível 3) }\end{array}$ & Área $\mathbf{K m}^{2}$ \\
\hline 112 & Tecido urbano descontínuo & 1,12 \\
\hline 212 & Cultura temporárias de regadio & 1 \\
241 & Culturas temporárias e/ou pastagens associadas a culturas permanentes & 0,56 \\
\hline 312 & Florestas de resinosas & 1,63 \\
\hline 313 & Florestas mistas & 0,32 \\
\hline 322 & Matos & 0,53 \\
\hline 324 & Florestas abertas, cortes e novas plantações & 0,18 \\
\hline 332 & Vegetação esparsa & 0,01 \\
\hline 522 & Desembocaduras fluviais & 0,65 \\
\hline Total & & 6,01 \\
\hline
\end{tabular}


Analisando as modelações realizadas com a Corine Land Cover 2006 (figura 3 e tabela V), o impacto no ambiente ocorreria, para os níveis $>50 \mathrm{ppm}$, numa área de $1,63 \mathrm{~km}^{2}$ de florestas resinosas, $0,53 \mathrm{~km}^{2}$ de matos, $0,32 \mathrm{~km}^{2}$ de florestas mistas, $0,18 \mathrm{~km}^{2}$ de florestas aberta, 0,01 $\mathrm{km}^{2}$ de vegetação esparsa e $0,65 \mathrm{~km}^{2}$ de desembocaduras fluviais do Rio Minho. É importante referir que o Rio Minho e as suas margens se encontram integradas na Rede Natura 2000 pelo que qualquer dano no seu ecossistema representa uma agravante séria a nível ambiental. A exposição da vegetação a uma fuga acidental de cloro dar-se-á sobretudo através da dispersão do gás na atmosfera. A vegetação exposta poderá sofrer danos e diminuição da área foliar, diminuição nos níveis de clorofila e morte. (EPA, 2003)

\section{Conclusão}

A relação entre riscos e o território é complexa e apenas a análise detalhada dos locais e contextos específicos nos poderá ajudar a compreender a suas dinâmicas. 0 território não deve ser estudado sem se considerarem os perigos, vulnerabilidades e riscos que enfrenta e, por outro lado, nenhum risco pode ser avaliado sem ter em consideração a dimensão espacial (Queirós, 2009). No caso concreto do trabalho realizado, a integração entre o ALOHA e o ArcMap, complementados pela metodologia "Areal Weighting", permite estimar as potências consequências da fuga de cloro para a população e ambiente. Assim, poder-se-á concluir, através da análise dos resultados, que um acidente envolvendo o transporte de cloro poderá acarretar um elevado dano nas populações e no ambiente. Além da metodologia proposta, este estudo constitui-se também como instrumento de apoio à prevenção, resposta e mitigação de futuros acidentes, contribuindo para a sensibilização, formação e resposta de todos os agentes de proteção civil.

\section{Bibliografia}

Autoridade Nacional de Proteção Civil (2011) - Manual de intervenção em emergências com matérias perigosas - químicas, biológicas e radiológicas. Carnaxide, ANPC, p.313;

Chakraborty, J., Armstrong, M.P., (1996) - Using Geographic plume analysis to assess community vulnerability to hazardous accidents, Computers, Environment and Urban Systems. [Online] 19, 341-356. Disponível em: http://www.sciencedirect.com/science/article/pii/0198971595000186 [Acedido em 14 de Janeiro de 2013]

Evans, R.B. (2005) - Chlorine: State of the art. Lung, 183(3), 151-167

Margia, F. L. 2001 - Health Risks and Environmental Inequity: A Geographical Analysis of Accidental Releases of Hazardous Materials. The Professional Geographer. [Online] 53(3):422-34. Disponível em: http:// www.tandfonline.com/doi/abs/10.1080/00330124.2001.9628473\#.U9phzvldVCg [Acedido em 14 de Janeiro de 2013]

Queirós, M. (2009) - Riscos e Ordenamento do Território: Prometeus ou conhecimento e partilha. Planeamento Civil de Emergência, [Online] 21, 20-28. Disponível em: http://riskam.ul.pt/images/pdf/ artrevnac_2009_\%20riscos_ot_prometeus.pdf [Acedido em 14 de Janeiro de 2013]

Winder, C. (2001) - The toxicology of chlorine. Environmental Research, 85(2), 105-114.

U.S. Environmental Protection Agency (EPA) \& National Oceanic and Atmospheric Administration (NOAA) (2007) - ALOHA user manual, [Online] 195. Disponível em: http://www2.epa.gov/cameo/cameodownloading-installing-and-running-aloha [Acedido em 14 de Janeiro de 2013]

U.S. Enviromental Protection Agency (2003) - National Emission Standards for Hazardous Air Pollutants: Chlorine and Hydrichloric Acid Emissions from Chlorine Prodution, Federal Register, vol.(68). [7094770957] 